\title{
Rotational and Magnetic Corrections to the g-Mode Frequencies in a One-Dimensional MHD Model
}

\author{
M. S. Dvornikov ${ }^{1,2}$, T. I. Rashba ${ }^{1,3}$, and V. B. Semikoz ${ }^{1}$ \\ ${ }^{1}$ Pushkov Institute of Terrestrial Magnetism, Ionosphere and Radio Wave Propagation, \\ Russian Academy of Sciences, Troitsk, Moscow oblast, 142190 Russia \\ ${ }^{2}$ Department of Physics, University of Juväscylä, Juväscylä, Finland \\ ${ }^{3}$ Max Planck Institute of Physics (Werner Heisenberg Institute), Munich, Germany \\ Received August 13, 2007; in final form, September 22, 2007
}

\begin{abstract}
The central magnetic field and rotation of the solar radiative zone are responsible for corrections to the g-mode frequencies. Magnetogravitational spectra are calculated analytically in a simple onedimensional MHD model that goes beyond the WKB approximation and avoid any cusp resonances that trap the wave within the radiative zone in the presence of a weak magnetic background. The calculations are compared with spacecraft observations of the $1 \%$ frequency shifts for candidate g-modes found in the SOHO GOLF experiment. The magnetic correction is the main contribution for a strong magnetic field satisfying the approximation used. It is shown that a constant magnetic field of $700 \mathrm{kG}$ in the radiative zone provides the required frequency shift for the $n=-10 \mathrm{~g}$-mode. The rotational correction, which is due to the Coriolis force in the one-dimensional model used, is much less than a percent $\left(\alpha_{\Omega} \leq 0.003\right)$.

PACS numbers: 96.60. Jw, 96.60.Bn, 96.60.Hv, 96.60.Ly, 95.30.Qd

DOI: $10.1134 / \mathrm{S} 1063772908040082$
\end{abstract}

\section{INTRODUCTION}

Recent progress in searches for low-frequency oscillations of the solar surface that have revealed possible candidate $\mathrm{g}$ modes (the SOHO GOLF experiment [1]) and prospects for new spacecraft experiments aimed at investigating the internal solar structure [2] provide new capabilities for direct, detailed studies of the radiative zone and solar core, which cannot be studied using helioseismology and observations of high-frequency p modes.

The frequencies found in [1] are shifted by a relative amount $\delta \omega / \omega \sim 1 \%$ compared to the predictions of the seismic model of the Sun [3, 4], which neglects both the magnetic field and the solar rotation.

The effect exerted by the magnetic field of the radiative zone on the g-mode frequencies was studied in [5], where the frequency shifts were calculated using a simple one-dimensional (1D) MHD model. This model that neglects the solar rotation and determines the limits for the application of the magnetic perturbation theory, which, in turn, neglect absorption and reflection of the g modes by Alfvenic or cusp radiative-zone layers. This approach implies that $g$ modes appear at the solar surface; such 1D calculations agree well with more recent estimates of the magnetic shifts determined in the three-dimensional (3D) MHD model [6]. ${ }^{1}$

Correct treatments of the cusp (Alfvenic) resonances in the 1D model (see Section 3.2) simplify the problem and display some advantages over 3D calculations of the rotational and magnetic corrections to the g-mode spectra. In the $3 \mathrm{D}$ model, these corrections can be calculated using perturbation theory disregarding the MHD resonances and using the eigenfunctions (horizontal and vertical displacements $\xi_{h, r}^{(n, l)}$ ) calculated for a solar seismic model, neglecting the external magnetic field and rotation of the star. Since the eigenfunctions of the $1 \mathrm{D}$ problem (the vertical velocities $v_{z}^{\left(n, k, \Omega, B_{0}\right)}(z)=$ $\left.-i \omega\left(n, k, \Omega, B_{0}\right) \xi_{z}^{\left(n, k, \Omega, B_{0}\right)}(z)\right)$ represent exact ana-

\footnotetext{
${ }^{1}$ In particular, this $3 \mathrm{D}$ model predicts that the $g_{10}^{1}$ mode frequency shift is of the order of $\delta \omega / \omega \sim 1 \%$, and can be attributed to the strong radiative-zone magnetic field $B=$ 2.5 MG, which corresponds to $B=2.2 \mathrm{MG}$ in the $1 \mathrm{D}$ MHD model with the maximum applicable Brunt-Väisälä frequency of $N=2.8 \times 10^{-3} \mathrm{rad} / \mathrm{s}=\operatorname{const}\left(\nu_{N}=N / 2 \pi=\right.$ $440 \mu \mathrm{Hz}$ ) instead of $N=10^{-3} \mathrm{rad} / \mathrm{s}=$ const used in Fig. 4 of [5]. Note that, in contrast to the analytical $1 \mathrm{D}$ calculations, which all used $N=$ const, the $N(r)$ profile in the numerical $3 \mathrm{D}$ model is given by the codes of the seismic model of the Sun $[3,4]$.
} 
lytical solutions, they depend on both the magnetic field and stellar rotation.

Here, we continue the analysis of analytical MHD spectra for internal helioseismic waves in the 1D model, going beyond the WKB approximation and taking into account both the magnetic field and solar rotation. Note that the 3D solar seismic-model calculations are able to include solar rotation only in the short-wave ( $L \ll c_{s}^{2} / g$ ) WKB approximation [7].

Note also that, due to the simplification of the topology in the 1D model, we lose the effect of the stellar rotation relative to the observer, with this effect being described by the azimuthal splitting of the $3 \mathrm{D}$ frequency $\sim m \Omega$. However, even for rigid-body (homogeneous) rotation, the Coriolis force arising in a frame rotating with the star leads to additional frequency splitting, which remains in the $1 \mathrm{D}$ model. The goal of our work is to estimate these effects calculated for magnetized plasmas in the 1D model using the exact analytical solutions, and going beyond perturbation theory (used in the 3D model) or the WKB approximation.

The contents of this article are arranged as follows. Section 2 describes the MHD model for ideal plasmas, with all the approximations discussed in Section 2.1. In Section 2.2, we estimate the effect of the rotation of the differential radiative zone and apply the approximation of rigid-body rotation of the radiative zone. In Section 3, we linearize the complete set of MHD equations and derive the main equation for the $z$ component of the perturbation velocity $v_{z}(z)$, which determines all other MHD perturbations, including those of the magnetic field. It is this component that results in the Doppler shift of the optical frequencies detected in helioseismology experiments.

Section 3.1 examines the non-magnetic case taking into account only the solar rotation. In Section 3.2, we derive a simplified form of the main equation for $v_{z}(z)$ in the limit of comparatively weak magnetic fields, making it possible to avoid radiativezone MHD resonances (cusp or Alfvenic). Note that such resonances were studied in [8-10]. Section 3.2 presents the exact analytical g-mode spectrum obtained using the magnetic-field perturbation method, taking into account the magnetic field in the radiative zone and the solar rotation. Section 4 discusses the results obtained.

\section{BASIC IDEAL MHD EQUATIONS}

We will use the following MHD equations. The law of mass conservation for the total density takes the form

$$
\frac{\partial \bar{\rho}}{\partial t}+\nabla \cdot(\bar{\rho} \mathbf{v})=0
$$

where the total density contains two terms, corresponding to the background density and the perturbation $\bar{\rho}=\rho_{0}+\rho^{\prime}$.

In a frame corotating with the Sun $\left(\mathbf{v}_{0}=0\right)$, the conservation of momentum can be expressed

$$
\begin{gathered}
\frac{\partial \mathbf{v}}{\partial t}+(\mathbf{v} \nabla) \mathbf{v}+2(\boldsymbol{\Omega} \times \mathbf{v}) \\
=-\frac{1}{\bar{\rho}} \nabla\left(p+\frac{H^{2}}{8 \pi}\right)+\frac{1}{8 \pi \bar{\rho}}(\mathbf{H} \nabla) \mathbf{H}+\mathbf{g} \\
-\boldsymbol{\Omega} \times(\boldsymbol{\Omega} \times \mathbf{r})+\mathbf{r} \times\left[\frac{\partial \boldsymbol{\Omega}}{\partial t}+(\mathbf{v} \nabla) \boldsymbol{\Omega}\right],
\end{gathered}
$$

where the total magnetic field $\mathbf{H}=\mathbf{B}_{0}+\mathbf{B}^{\prime}$ contains the background magnetic field $\mathbf{B}_{0}$ and the perturbation $\mathbf{B}^{\prime}, \mathbf{g}$ is the gravitational acceleration, and $\boldsymbol{\Omega}$ is the rotational frequency.

The ideal MHD Faraday equation takes the form

$$
\frac{\partial \mathbf{H}}{\partial t}=(\mathbf{H} \cdot \nabla) \mathbf{v}-(\mathbf{v} \cdot \nabla) \mathbf{H}-\mathbf{H} u,
$$

where $u=\nabla \cdot \mathbf{v} \neq 0$ is the gas compressibility.

Finally, the set of nonlinear MHD equations is completed by the conservation of entropy:

$$
\frac{\partial}{\partial t}\left(\frac{\bar{p}}{\bar{\rho}^{\gamma}}\right)+(\mathbf{v} \cdot \nabla)\left(\frac{\bar{p}}{\bar{\rho}^{\gamma}}\right)=0
$$

where $\bar{p}=p_{0}+p^{\prime}$ is the total gas pressure and $\gamma=$ $c_{p} / c_{V}$ is the ratio of specific heats $(\gamma=5 / 3$ for the hydrogen plasma).

Note that, when

$$
-\frac{1}{\rho_{0}} \nabla\left(p_{0}+\frac{B_{0}^{2}}{8 \pi}\right)+\mathbf{g}-\boldsymbol{\Omega} \times(\boldsymbol{\Omega} \times \mathbf{r})=0,
$$

the contribution of the centrifugal force (the last term) is negligible for equilibrium states of the Sun $\left(\sim 2 \times 10^{-5}\right)$.

\subsection{Assumptions of the Rectangular 1D Model}

To determine the spectra of the seismic waves taking into account the magnetic field and rotation of the radiative zone, we must adopt a number of assumptions. We will describe these assumptions as they are used, in order to clarify the consequences of each. Belowm, we present a complete list of the assumptions used, for convenience in our subsequent references to them.

1. The ideal MHD description neglects the contributions of the thermal conductivity and viscosity to energy losses and assumes an infinite Ohmic conductivity.

2. We linearize the MHD equations near the equilibrium background, which is a time-independent background configuration given by (5).

ASTRONOMY REPORTS Vol.52 No.4 2008 
3. We assume that the fluctuations are adiabatic, with their contributions to the heat source being negligible $Q^{\prime}=0$.

4. The gas is assumed to be ideal and completely ionized. This means that the ratio of specific heats $\gamma=c_{p} / c_{V}$ is time-independent. For the numerical calculations, we use $\gamma=5 / 3$, which corresponds to hydrogen plasma.

5. We use the Cowling approximation of negligible perturbations of the gravitational potential, i.e., $\phi^{\prime}=0$.

6. Rigid-body rotation is assumed in the radiative zone.

7. We use the Cartesian coordinates $x, y$, and $z$, where $z$ corresponds to the radial direction inside the Sun (see Fig. 1). The background quantities depend only on $z$. This means that the local acceleration of gravity $\mathbf{g}$ is in the $-z$ direction. We also assume a constant uniform magnetic background field $\mathbf{B}_{0}$ directed along the $x$ axis. The rotation (angular velocity vector $\boldsymbol{\Omega}$ ) is in the $y$ direction, which is perpendicular to the ecliptic (the $x 0 z$ plane). Far from the center of the Sun, this 1D configuration corresponds to a large-scale toroidal 3D magnetic field in the radiative zone.

8. The background density profile is assumed to be exponential, $\rho_{0}=\rho_{c} \exp (-z / H)$, with constant $\rho_{c}$ and $H$. Hydrostatic equilibrium determines the background profiles of the thermodynamic quantities, so that $\gamma$ is constant.

9. The Brunt-Väisälä frequency is assumed to be zero in the convective zone and to be a non-zero constant in the radiative zone.

The last assumption is applicable to the real mass and density profiles of the standard solar model if the $z$ direction is radial, taking into account deep radiative zone layers and neglecting the narrow layer at the center of the Sun $(z \ll H)$, where $N(z) \rightarrow 0$ (see, for example, [8]). A constant $\gamma$ is also expected in this region, since a strongly ionized plasma satisfies the ideal gas equation of state well. This geometry is reasonable when we examine regions far from the solar center. The fact that $\mathbf{B}_{0}$ is slowly varying in this region is important, and also the fact that this vector is perpendicular to both $\mathbf{g}$ and to the gradients of all the background quantities $\nabla \rho_{0}, \nabla p_{0}$, etc.

It was proposed in [8] that this one-dimensional model can be completely described analytically, in contrast to the three-dimensional case.

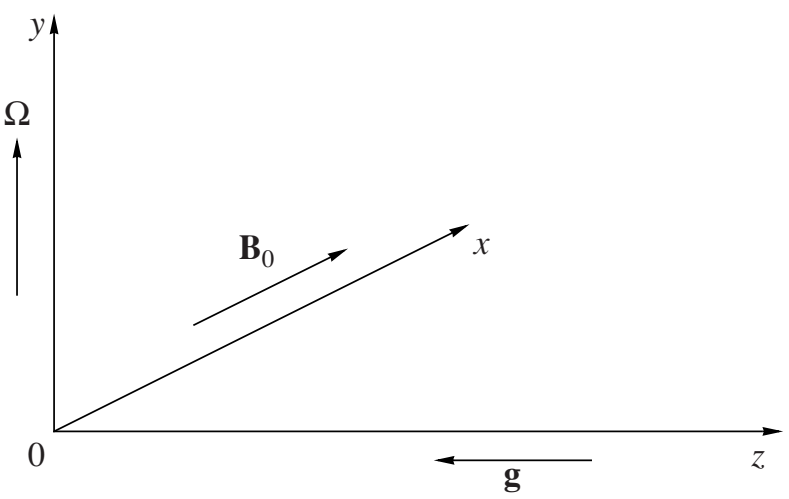

Fig. 1. Rectangular 1D geometry.

\subsection{Differential Rotation}

In three dimensions (spherical coordinates) with $\boldsymbol{\Omega}=\Omega \cos \theta \mathbf{e}_{r}-\Omega \sin \theta \mathbf{e}_{\theta}$ and with no angular acceleration $(\partial \Omega / \partial t=0)$, the last term of $(2)$, $-r \sin \theta(\mathbf{v} \nabla) \Omega \mathbf{e}_{\varphi}$, becomes much smaller than the contribution of the Coriolis force on the left-hand side if

$$
r \sin \theta\left(v_{r} \frac{\partial \Omega}{\partial r}+\frac{v_{\theta}}{r} \frac{\partial \Omega}{\partial \theta}\right) \ll 2 \Omega\left(\sin \theta v_{r}+\cos \theta v_{\theta}\right)
$$

or

$$
r \frac{\partial \Omega}{\partial r} \ll 2 \Omega, \quad \tan \theta \frac{\partial \Omega}{\partial \theta} \ll 2 \Omega .
$$

It is clear that these conditions can be satisfied in the radiative zone (for nearly rigid-body rotation), while this last inequality is valid near the equator in the convective zone. ${ }^{2}$ The SOHO solar-rotation profile presented in the right panel in Fig. 1 of [11] shows that the first condition of $(6)$ is satisfied with an accuracy of $\sim \Delta \Omega / \Omega \sim 50 / 400=0.125 \ll 1$, at least at low and middle latitudes.

In our rectangular geometry, the corresponding conditions

$$
z \frac{d \Omega}{d z} \ll 2 \Omega, \quad x \frac{d \Omega}{d z} \ll 2 \Omega \frac{v_{x}}{v_{z}}
$$

are analogous to (6), with the velocity ratio of the second inequality $(7)$ exceeding unity $\left(v_{x} / v_{z}>1\right)$, especially for MHD resonances, when $v_{x} \gg v_{z}$.

We, therefore, assume that $\Omega=$ const, thereby neglecting angular acceleration and differential rotation and discarding the last line in (2).

\footnotetext{
${ }^{2}$ The equator $\theta=\pi / 2$ is inclined by $7^{\circ}$ to the ecliptic latitude $\vartheta=0^{\circ}(\theta=\pi / 2)$ only in the limit when $\partial \Omega /\left.\partial \theta\right|_{\theta=\pi / 2} \rightarrow 0$.
} 


\section{LINEAR IDEAL MHD EQUATIONS FOR THE ROTATING SUN}

Assuming plane waves propagating along the background magnetic field $\left(\sim e^{-i\left(\omega t-k_{x} x\right)}\right)$, we can reduce the complete MHD system (1)-(4) to the following eight linearized equations:

$$
\begin{gathered}
\rho^{\prime}=\frac{v_{z}}{i \omega} \frac{d \rho_{0}}{d z}+\frac{\rho_{0}}{i \omega}\left(\frac{\partial v_{y}}{\partial y}+\frac{\partial v_{z}}{\partial z}\right)+\frac{\rho_{0} v_{x}}{V}, \\
P=\rho_{0} V v_{x}+\frac{B_{0} B_{x}^{\prime}}{4 \pi}-\frac{2 \rho_{0} \Omega v_{z}}{i k_{x}}, \\
\frac{\partial P}{\partial y}=i \rho_{0} \omega v_{y}+\frac{i k_{x} B_{0}}{4 \pi} B_{y}^{\prime}, \\
\frac{\partial P}{\partial z}=i \rho_{0} \omega v_{z}+\frac{i k_{x} B_{0}}{4 \pi} B_{z}^{\prime}+2 \rho_{0} \Omega v_{x}-\rho^{\prime} g, \\
B_{x}^{\prime}=\frac{B_{0}}{i \omega}\left(\frac{\partial v_{y}}{\partial y}+\frac{\partial v_{z}}{\partial z}\right), \\
B_{y}^{\prime}=-\frac{B_{0}}{V} v_{y}, \\
\rho^{\prime}=\frac{1}{c_{s}^{2}}\left(P-\frac{B_{0}^{\prime} B_{x}}{4 \pi}\right)+\frac{B_{z}}{V} v_{z}, \\
\gamma(\gamma-1) \\
\frac{B^{\prime}}{\gamma} \frac{d \rho_{0}}{d z}
\end{gathered}
$$

where $V=\omega / k_{x}$ is the phase velocity of the MHD oscillations required, $P=p^{\prime}+B_{0} B_{x}^{\prime} / 4 \pi$ the total pressure perturbation in the linear approximation, $c_{s}=$ $\sqrt{\gamma g H}$ the speed of sound, $H=0.1 R_{\odot}$ the characteristic scale of the background density variations $\rho_{0}=\rho_{c} e^{-z / H}$, and $\rho_{c}=150 \mathrm{~g} / \mathrm{cm}^{3}$ the density at the center of the Sun.

Let us derive $P-B_{0} B_{x}^{\prime} / 4 \pi$ from (9). Then equating (8) and (15), we express $v_{x}$ through $v_{y, z}$ as

$$
v_{x}=\frac{c_{s}^{2} V}{V^{2}-c_{s}^{2}}\left[\frac{1}{i \omega}\left(\frac{\partial v_{y}}{\partial y}+\frac{\partial v_{z}}{\partial z}\right)\right.
$$

$$
\left.+\frac{v_{z}}{i \omega \gamma \rho_{0}} \frac{d \rho_{0}}{d z}+\frac{2 \Omega v_{z}}{i k_{x} c_{s}^{2}}\right] .
$$

Equation (10) shows that we can take $v_{y}=B_{y}^{\prime}=$ $\partial / \partial y=0$ for the geometry considered; i.e., the radiative zone material is homogeneous along the rotational axis ( $y$ axis). This enables us to derive the main second-order differential equation

$$
\begin{gathered}
\zeta(1-\zeta) \frac{d^{2} v_{z}}{d \zeta^{2}}-\zeta \frac{d v_{z}}{d \zeta} \\
+k^{2}\left\{1+\zeta^{-1}\left[\frac{N^{2}}{\omega^{2}}-1+\frac{\omega^{2}-4 \Omega^{2}}{k_{x}^{2} c_{s}^{2}}\right.\right. \\
\left.\left.+\frac{2 H \Omega}{V k^{2}}\left(\frac{2}{\gamma}-1\right)\right]\right\} v_{z}=0
\end{gathered}
$$

from (11) using (9) for the pressure $P$ and (12) for $B_{x}^{\prime}$, together with the expression for $v_{x}$ obtained from (16). Here, we introduce the Brunt-Väisälä frequency $N$ :

$$
N^{2}=\frac{c_{s}^{2}}{H^{2}} \frac{\gamma-1}{\gamma^{2}}=\frac{g}{H} \frac{\gamma-1}{\gamma} .
$$

This frequency normalizes the compressibility $a_{1}=$ $\omega^{2} / k_{x}^{2} c_{s}^{2}=0.24(\omega / N)^{2} / K^{2}$, where the coefficient $(\gamma-1) / \gamma^{2}=0.24$ is calculated for the hydrogen plasma with $\gamma=5 / 3 \quad\left(a_{1} \rightarrow 0\right.$ in incompressible gases); $K=k_{x} H$ is the dimensionless wave number. The argument $\zeta=\left(1-a_{1}\right) \xi$ is determined by the background magnetic field $B_{0} ; \xi=\xi_{0} e^{z / H}$ and $\xi_{0}=k_{x}^{2} v_{A 0}^{2} / \omega^{2}$, where $v_{A 0}[\mathrm{~cm} / \mathrm{s}]=B_{0} / 43.4 \mathrm{G}$ is the Alfven velocity at the solar center for the central density used.

Following [8], we can find the desired solution of this equation using Gauss' equation for $v_{z}=\zeta^{\sigma} Y(\zeta)$,

$$
\begin{gathered}
v_{z}(\zeta)=D_{1} \zeta^{\sigma_{1}} F(a, b ; c ; \zeta) \\
+D_{2} \zeta^{1-\sigma_{1}-\gamma^{-1}} F(1+a-c, 1+b-c ; 2-c ; \zeta),
\end{gathered}
$$

where $F(a, b ; c ; \zeta)$ is a hypergeometric Gaussian and

$$
\sigma_{1,2}=\frac{1}{2} \pm \frac{1}{2} \sqrt{1+4 K^{2}\left[\frac{N^{2}}{\omega^{2}}-1+\frac{\omega^{2}-4 \Omega^{2}}{k_{x}^{2} c_{s}^{2}}+\frac{2 \Omega}{\omega k}\left(\frac{2}{\gamma}-1\right)\right]}
$$

determine its indices (the upper sign corresponds to $\left.\sigma_{1}\right)$, namely $a=\sigma_{1}+K, b=\sigma_{1}-K$, and $c=a+b$.

Using reasonable boundary conditions (see, for example, [8]), we can determine the MHD dis- persion equation for magnetogravitational waves $\omega\left(K, B_{0}, \Omega\right)$, which will be expressed through the hypergeometric functions of (18) for the corresponding point (at the center of the Sun, $z=0$ and 
$\left.\zeta=\zeta_{0}=\left(1-a_{1}\right) \xi_{0}\right)$. However, the analysis of this equation is rather complicated. Below, we consider some simplified versions of (17) that enable us to obtain some analytical spectra for the radiative zone.

\subsection{The Limit of Zero Magnetic Field}

For the limit $\zeta=\left(1-a_{1}\right) \xi \rightarrow 0, B_{0} \rightarrow 0$, we rearrange the equation in the form

$$
\begin{gathered}
\frac{d^{2} \Psi}{d z^{2}}+\left\{\frac{\omega^{2}-4 \Omega^{2}}{c_{s}^{2}}-\frac{1}{4 H^{2}}\right. \\
\left.+k_{x}^{2}\left[\frac{N^{2}}{\omega^{2}}-1+\left(\frac{2}{\gamma}-1\right) \frac{2 \Omega}{\omega k_{x} H}\right]\right\} \Psi=0
\end{gathered}
$$

using the transformation $v_{z}=e^{z / 2 H} \Psi(z)$, the substitution $\zeta \frac{d}{d \zeta}=H \frac{d}{d z}$, and (17). In the absence of rotation $(\Omega=0)$, this equation is quite similar to $(7.90)$ of [12], which describes the 3D oscillations:

$$
\frac{d^{2} \Psi}{d r^{2}}+\left[\frac{\omega^{2}}{c_{s}^{2}}-\frac{1}{4 H^{2}}+k_{h}^{2}\left(\frac{N^{2}(r)}{\omega^{2}}-1\right)\right] \Psi=0 .
$$

When $\Omega=0$, we rewrite (20) in the form

$$
\frac{d^{2} \Psi}{d z^{2}}+\frac{\beta_{0}^{2}}{4 H^{2}} \Psi(z)=0
$$

for the radiative zone, where $\beta_{0}=\sqrt{4 K^{2}\left(N^{2} / \omega^{2}-1\right)-1+4 K^{2} a_{1}}$, and in the form

$$
\frac{d^{2} \Psi}{d z^{2}}-\frac{\Gamma_{0}^{2}}{4 H^{2}} \Psi(z)=0,
$$

for the convective zone, where $N_{C Z}=0$ and $\beta_{0} \rightarrow$ $i \Gamma_{0}=i \sqrt{4 K^{2}\left(1-a_{1}\right)+1}$. We then join the solutions at the upper boundary of the radiative zone $z=z_{R Z}$ to obtain the g-mode spectrum in the $1 \mathrm{D}$ model [5]:

$$
\frac{\beta_{0} z_{R Z}}{2 H}+\pi n=\operatorname{arccot}\left(-\frac{\Gamma_{0}}{\beta_{0}}\right)
$$

where $n=-1,-2,-3, \ldots$ The analogous solution of (20) for the rotating Sun $(\Omega \neq 0)$ yields the spectrum

$$
\frac{\beta_{\Omega} z_{R Z}}{2 H}+\pi n=\operatorname{arccot}\left(-\frac{\Gamma_{\Omega}}{\beta_{\Omega}}\right),
$$

where

$$
\beta_{\Omega}=\sqrt{0.96\left[\left(\frac{\omega}{N}\right)^{2}-\left(\frac{2 \Omega}{N}\right)^{2}\right]-1+4 k^{2}\left[\frac{N^{2}}{\omega^{2}}-1+\frac{1}{5 k}\left(\frac{2 \Omega}{N}\right)\left(\frac{N}{\omega}\right)\right]}
$$

and

$$
\Gamma_{\Omega}=\sqrt{4 k^{2}\left[1-\frac{\omega^{2}-4 \Omega^{2}}{k_{x}^{2} c_{s}^{2}}-\frac{1}{5 K}\left(\frac{2 \Omega}{\omega}\right)\right]+1},
$$

where $n=-1,-2, \ldots$ We can neglect $\Omega^{2}$ in (24) and (25), since $2 \Omega / N=2 \times 10^{-3}$ is small compared to the frequencies revealed in the GOLF [1] experiment $(1>\omega / N \geq 0.2)$ and considered in our work.

A rough estimate for the splitting of the rotational frequency $\omega \rightarrow \omega-\Delta \omega$ due to the Coriolis force when $\omega \leq N$ can be obtained from (24) taking into account the shift $N^{2} / \omega^{2} \rightarrow\left(N^{2} / \omega^{2}\right)(1+2 \Delta \omega / \omega)$ :

$$
\begin{gathered}
\left|\alpha_{\Omega}\right| \equiv \frac{\Delta \omega}{\omega}=\frac{1}{10 K}\left(\frac{2 \Omega}{N}\right)\left(\frac{\omega}{N}\right) \\
=\frac{1}{5 K} 10^{-3}\left(\frac{\omega}{N}\right) .
\end{gathered}
$$

Consequently, this frequency shift is negligible for $v_{z}$ waves with $K>1$ propagating in the plane of the ecliptic $(\mathrm{g} \perp \boldsymbol{\Omega}), \alpha_{\Omega} \sim 10^{-4}$.

Figure 2 shows the $\mathrm{g}$ spectrum $\omega(n, K)$ taking into account the solar rotation $\Omega$ determined by the dispersion equation (23) for several modes, with $n=-1,-5,-10$. Figure 3 presents the g-mode frequency shifts $\alpha_{\Omega}(K)=\left(\omega-\omega_{0}\right) / \omega_{0}$ as functions of the transverse wave number $K$ and neglecting the 


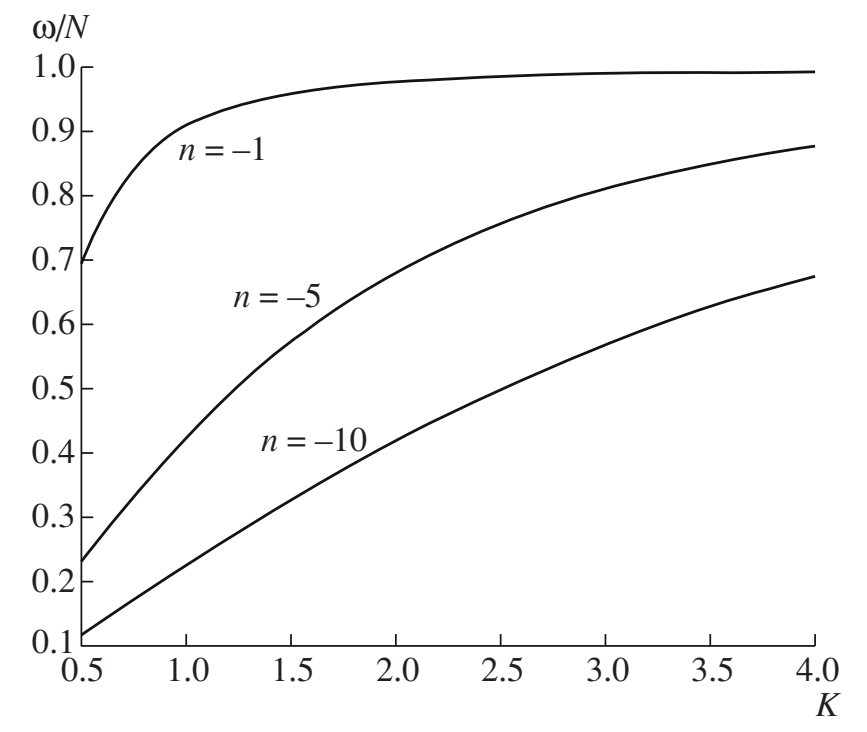

Fig. 2. The $g$ spectrum as a function of the wave vector $K$ for several modes $(n=-1,-5,-10)$ of the rotating Sun.

magnetic field in the radiative zone $\left(\mathbf{B}_{0}=0\right)$. In this case, the frequency $\omega_{0}$ corresponds to an absence of rotation $\left(\Omega=B_{0}=0\right)$. Note also that the rotational correction due to the Coriolis force increases when $K$ decreases, especially for the highest frequency $n=-1$. This behavior agrees with that in the threedimensional case (see below).

\subsection{Magnetic and Rotational Corrections to the $g$ Mode Spectrum}

To find the $\mathrm{g}$ mode spectra taking into account the magnetic field, we define the coefficient of the second derivative in (17) to be $1-\zeta$, where $\zeta=v_{A}^{2}(1-$ $\left.a_{1}\right) / V^{2}$.

Let us examine the magnetic fields in the framework of perturbation theory. In this case, the Alfven speed is much lower than the phase speed, namely $v_{A}^{2}=v_{A 0}^{2} e^{z / H} \ll V^{2}=\omega^{2} / k_{x}^{2}=$ $\left[N^{2} H^{2} / K^{2}\right](\omega / N)^{2} ; \zeta \ll 1$; and no MHD resonances $(\zeta=1)$ occur in the radiative zone [5, Fig. 2]. The magnetic field for which the perturbation theory is applicable, that is, for which the maximum Alfven speed remains low $\left(v_{A}^{2}\left(0.7 R_{\odot}\right) \ll V^{2}\right)$, satisfies the relation

$$
B_{0} \ll \frac{28}{K}\left(\frac{\omega}{N}\right) M G .
$$

This inequality determines the admissible magnetic fields in order for the perturbation theory to be applicable. It can be verified that this relation is satisfied well for the magnetic fields considered here, and for modes reaching $n=-10$.

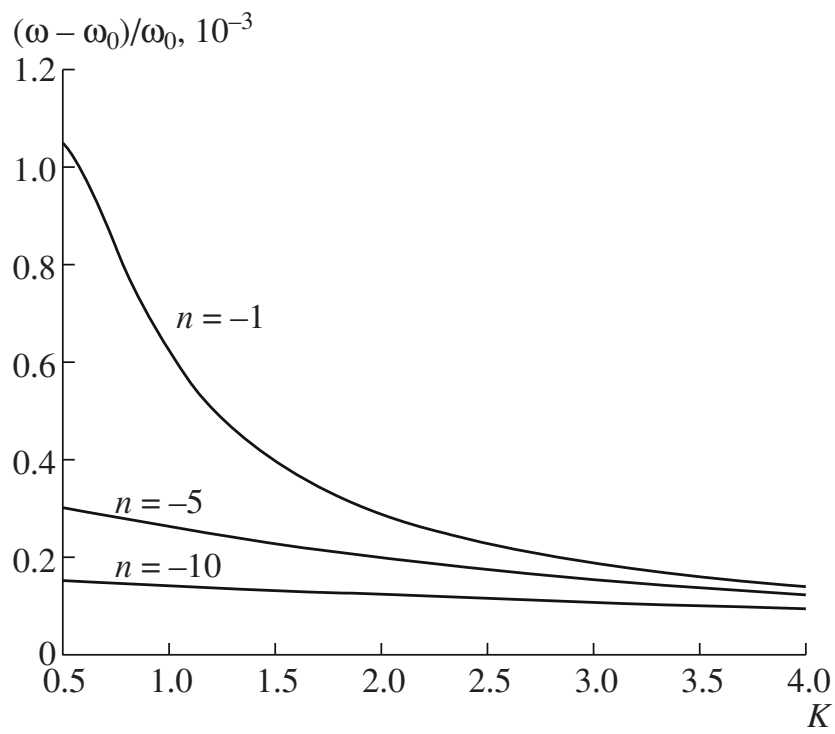

Fig. 3. Relative rotational frequency shift as a function of the wave vector $K$ for the $n=-1,-5,-10 \mathrm{~g}$ modes.

In the absence of MHD resonances, we can assume that

$$
v_{z}=\left[1-\frac{v_{A}^{2}}{V^{2}}\left(1-\frac{V^{2}}{c_{s}^{2}}\right)\right]^{-1 / 2} e^{z / 2 H} \Psi(z),
$$

and determine the magnetic correction $O\left(v_{A}^{2} / V^{2}\right)$ for (20) from the main equation (17):

$$
\frac{d^{2} \Psi}{d z^{2}}+\left(\frac{\beta_{\Omega}^{2}}{4 H^{2}}+k_{B}^{2}(\Omega)\right) \Psi=0,
$$

where $\beta_{\Omega}$ is determined by (24), and the correction to the wave vector $k_{B}^{2}(\Omega)$ satisfies the formula

$$
\begin{gathered}
k_{B}^{2}(\Omega)=k_{x}^{2}\left(1-a_{1}\right) \frac{v_{A}^{2}}{V^{2}}\left[\frac{N^{2}}{\omega^{2}}+a_{1}\right. \\
\left.-\left(\frac{2 \Omega}{N}\right)^{2}\left(\frac{0.24}{K^{2}}\right)+\frac{1}{5 K}\left(\frac{2 \Omega}{N}\right)\left(\frac{N}{\omega}\right)\right] .
\end{gathered}
$$

Introducing the coefficient

$$
\begin{gathered}
A_{0}=\left(1-a_{1}\right) \frac{v_{A 0}^{2}}{V^{2}}\left[\frac{N^{2}}{\omega^{2}}+a_{1}\right. \\
\left.-\left(\frac{2 \Omega}{N}\right)^{2}\left(\frac{0.24}{K^{2}}\right)+\frac{1}{5 K}\left(\frac{2 \Omega}{N}\right)\left(\frac{N}{\omega}\right)\right]
\end{gathered}
$$

and using the substitution $2 s=z / H+\ln \left(4 K^{2} A_{0}\right)$, we can rewrite (27) in the form

$$
\frac{d^{2} \Psi}{d s^{2}}+\left(\beta_{\Omega}^{2}+e^{2 s}\right) \Psi(s)=0 .
$$

This equation has a general solution expressed through the Bessel functions of the first kind 
$\Psi(s)=C_{1} J_{i \beta_{\Omega}}\left(e^{s}\right)+C_{2} J_{-i \beta_{\Omega}}\left(e^{s}\right)$, where $C_{1,2}$ are constants. Following the treatment of [5], we find a solution for the radiative zone using the boundary condition for the center of the Sun $\Psi(z=0)=0$ ( since $v_{z}(0)=0$ ). Thus, we eliminate one constant, in order to obtain a solution of (30) in the radiative zone solution $\left(0 \leq z \leq z_{R Z}\right)$

$$
\begin{aligned}
& \Psi_{R Z}(z)=C_{R Z}\left[J_{i \beta_{\Omega}}\left(2 K A_{0}^{1 / 2} e^{z / 2 H}\right)\right. \\
& \left.-\frac{J_{i \beta_{\Omega}}\left(2 K A_{0}^{1 / 2}\right)}{J_{-i \beta_{\Omega}}\left(2 K A_{0}^{1 / 2}\right)} J_{-i \beta_{\Omega}}\left(2 K A_{0}^{1 / 2} e^{z / 2 H}\right)\right] .
\end{aligned}
$$

In the convective zone, the Brunt-Väisälä frequency $N=0$. Therefore, we arrive at the same solution as for the isotropic case, namely, $\Psi_{C Z}(z)=$ $C_{C Z} \exp \left(-z \Gamma_{\Omega} / H\right)$, where $\Gamma_{\Omega}$ obeys (25). Thus, we neglect the effects of magnetic fields in the convective zone (see the comments concerning formula (25) of [5]). Since the Bessel function arguments are small, we can retain only the first terms of the Maclaurin expansion.

Equating the logarithmic derivatives of the solutions $\Psi_{R Z}$ and $\Psi_{C Z}$ at the upper point of the radiative zone $z=z_{R Z}$, we obtain the dispersion equation for the case $B_{0} \neq 0$, generalizing our previous equation (see (27) in [5]) for rigid-body rotation of the solar radiative zone:

$$
\begin{gathered}
\beta_{\Omega}\left(1+\frac{2 \kappa_{R Z}^{2}}{1+\beta_{\Omega}^{2}}\right) \\
\times\left[1-\beta_{\Omega} \frac{2 \kappa_{R Z}^{2}}{1+\beta_{\Omega}^{2}} \frac{1}{\sin \left(\beta_{\Omega} z_{R Z} / H\right)}\right] \cot \left(\beta_{\Omega} \frac{z_{R Z}}{H}\right) \\
-\frac{2 \kappa_{R Z}^{2}}{1+\beta_{\Omega}^{2}}=-\Gamma_{\Omega},
\end{gathered}
$$

where $\kappa_{R Z}=K A_{0}^{1 / 2} e^{z_{R Z} / 2 H}$ and the coefficients $A_{0}$, $\beta_{\Omega}$, and $\Gamma_{\Omega}$ are given by (29), (24), and (25), respectively.

This relation is our basic equation determining the $g$ mode spectrum $\omega_{B}(K)$ in the one-dimensional MHD model, including the solar rotation. Figure 4 presents the frequency splitting due to the Coriolis force for a magnetic field of $B_{0}=700 \mathrm{kG}$ for the $n=$ $-1,-5,-10 \mathrm{~g}$-modes (dashed curves) as functions of the transverse wave vector $K$.

\section{DISCUSSION}

As expected, the contribution of the Coriolis force to the $\mathrm{g}$ mode frequency shift is small, less than $1 \%\left(\alpha_{\Omega}<0.003\right)$, even for the radial index $n=-1$. Figure 4 shows the corrections to the magnetic shift $\alpha_{B}$ including the solar rotation $(\Omega \neq 0)$. Figure 4 compares two curves (for each frequency) calculated

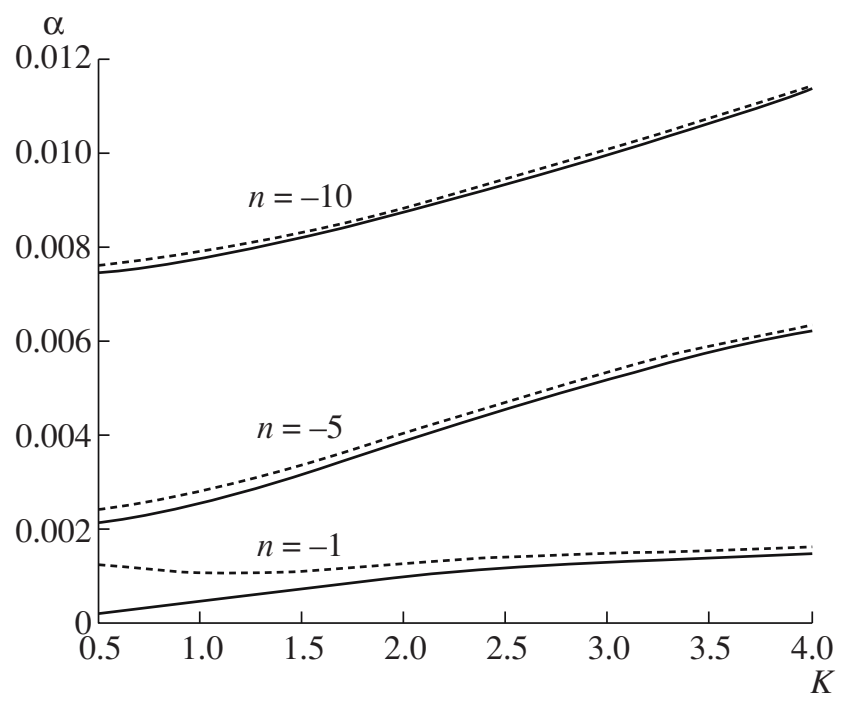

Fig. 4. Relative frequency shift due to the rotation and a magnetic field of $B_{0}=700 \mathrm{kG}$ as a function of the wave vector $K$ for the $n=-1,-5,-10$ modes, both neglecting (solid curves) and including the rotation (dashed curves).

for a central magnetic field of $B_{0}=700 \mathrm{kG}$ : the solid curves show the frequency shifts $\alpha_{B}(K)=\left(\omega_{B_{0}}-\right.$ $\left.\omega_{0}\right) / \omega_{0}$ for the previous results [5] neglecting the rotation $(\Omega=0)$, and the dashed curves the shifts $\alpha_{\Omega}(K)=\left(\alpha_{B}-\omega_{0}\right) / \omega_{0}$ simultaneously taking into account the magnetic field and the Coriolis force.

There is no azimuthal number $m$ in our onedimensional MHD model, and the transverse wave number $K$ is similar to an angular mode index $l$ (the Legendre number); a decrease in this wave number results in an increase in the Coriolis contribution, which agrees well with the effect of the 3D Coriolis force (see Fig. 4).

For an analytical treatment of the one-dimensional model, we must use a rough approximation of the homogeneous Brunt-Väisälä frequency $N(z)$ assuming $N=$ const, assuming, as in [5], this frequency to be $N=10^{-3} \mathrm{rad} / \mathrm{s}$. This is lower than the maximum Brunt-Väisälä frequency of $N=2.8 \times 10^{-3} \mathrm{rad} / \mathrm{s}$ (a cycle frequency of $\nu_{N}=N / 2 \pi=440 \mu \mathrm{Hz}$ [12]) by a factor of 2.8. If we adopt the maximum Brunt-Väisälä frequency, we should obtain a frequency shift that is lower by a factor of $(2.8)^{2}=7.84$ for the same magnetic field of $B_{0}=700 \mathrm{kG}$. On the other hand, using a constant Brunt-Väisälä frequency of $N=2.8 \times$ $10^{-3} \mathrm{rad} / \mathrm{s}$ yields the same magnetic shift of about $1 \%$ (allowing for the Coriolis force) for a stronger (by a factor of 2.8) radiative-zone magnetic field. For example, the magnetic field would be about 2 MG for the proper frequency shift of the $n=-10 \mathrm{~g}$ mode. 
Thus, the magnetic fields resulting in a frequency shift of $\delta \omega / \omega \sim B^{2} / \omega^{2} \sim 1 \%$ prove to be fairly strong, especially for the experimentally observed high $\mathrm{g}$ mode frequencies, which approach the Brunt-Väisälä frequency. For example, the corresponding frequency shift for the $g_{3}^{2}$ mode $(\nu=$ $222.46 \mu \mathrm{Hz}$ ) requires a strong radiative-zone magnetic field, $B \geq 8 \mathrm{MG}$ [6], which exceeds established limits for the central magnetic field. In particular, the flattening by $\sim 10^{-5}$ at the solar poles imposes the constraint $B<7$ MG [13]. This seeming contradiction can be understood if the observed frequency shift of the $g_{3}^{2}$ mode is not due to the magnetic field or rotation, but instead to some interactions with other g-modes with frequencies near the Brunt-Väisälä frequency.

Other configurations of the three-vectors $\mathbf{g}, \boldsymbol{\Omega}$, and $\mathbf{B}_{0}$ that differ from that presented in Fig. 1 for our one-dimensional model could result in other magnetogravitational mode spectra that we have not considered here.

In conclusion, we note that one ultimate goal of all studies of the internal structure of the Sun is the construction of a complete three-dimensional MHD model for the solar evolution. However, the formulation of such a model is a problem that must be addressed in the distant future. We have taken a step toward this goal by presenting a qualitative one-dimensional MHD model describing the effects exerted by the magnetic field in the radiative zone field on the spectra of internal waves, taking into account the solar rotation.

\section{ACKNOWLEDGMENTS}

V.B. Semikoz thanks S. Turck-Chièze and A. Brun for initial discussions. This work was supported by the Academy of Finland (contract no. 108875), the Russian Science Support Foundation (M.S.D.), and a European Marie Curie International Incoming Fellowship (T.I.R).

\section{REFERENCES}

1. S. Turck-Chièze, R. A. Garcia, S. Couvidat, et al., Astrophys. J. 604, 455 (2004).

2. S. Turck-Chièze, Adv. Space Res. 37, 1569 (2006); astro-ph/0511126.

3. S. Turck-Chièze, S. Couvidat, A. G. Kosovichev, et al., Astrophys. J. 555, L69 (2001).

4. S. Couvidat, S. Turck-Chièze, and A. G. Kosovichev, Astrophys. J. 599, 1434 (2003).

5. T. I. Rashba, V. B. Semikoz, and J. W. F. Valle, Mon. Not. R. Astron. Soc. 370, 845 (2006).

6. T. I. Rashba, V. B. Semikoz, S. Turck-Chièze, and J. W. F. Valle, Mon. Not. R. Astron. Soc. 377, 453 (2007).

7. P. Kumar, S. Talon, and J.-P. Zahn, Astrophys. J. 520, 859 (1999).

8. C. P. Burgess, N. S. Dzhalilov, T. I. Rashba, et al., Mon. Not. R. Astron. Soc. 348, 609 (2004).

9. C. P. Burgess, N. S. Dzhalilov, M. Maltoni, et al., Astrophys. J. 588, L65 (2003).

10. C. P. Burgess, N. S. Dzhalilov, M. Maltoni, et al., J. Cosmol. Astropart. Phys. 01, 007 (2004).

11. S. Turck-Chièze et al., in Proceedings of 39-th ESLAB Symposium: Trends in Space Science and Cosmic Vision 2020, Noordwijk, The Netherlands, 2005 , Ed. by F. Favata, J. Sanz-Forcada, A. Gimenez, and B. Battrick, ESA SP-588 (European Space Agency, 2005); astro-ph/0510854.

12. J. Christensen-Dalsgaard, Lecture Notes on Stellar Oscillations (Inst. for Phys. and Astron., Theor. Astrophys. Center, Copenhagen, 2003), p. 145; http://astro.phys.au.dk/jcd/oscilnotes/.

13. A. Friedland and A. Gruzinov, Astrophys. J. 601, 570 (2004).

Translated by V. Badin 